\title{
Publisher's Note: Unusual magnetotransport from Si-square nets in topological semimetal HfSiS [Phys. Rev. B 95, 121109(R) (2017)]
}

Nitesh Kumar, Kaustuv Manna, Yanpeng Qi, Shu-Chun Wu, Lei Wang, Binghai Yan, Claudia Felser, and Chandra Shekhar

(Received 30 March 2017; published 6 April 2017)

DOI: 10.1103/PhysRevB.95.159903

This paper was published online on 27 March 2017 without the author corrections incorporated into the published article. The paper has been corrected as of 30 March 2017. The text is correct in the printed version of the journal. 